\title{
The Extension of Hypothesis in Propositional Logic
}

\author{
Qian Guo ${ }^{1}$ and An-Sheng Deng ${ }^{1}$ \\ ${ }^{1}$ Information Science and Technology College, Dalian Maritime University, 116026 Dalian, China
}

\begin{abstract}
The belief non-revision method can achieve a consistent hypothesis extension by limiting the process of reasoning. In propositional logic, several valuable properties of the hypothesis extension have been proved. The hypothesis extension is an infinite set. It includes some clauses. For improving the efficiency of the non-revision method, this paper proposed an algorithm of hypothesis extension in propositional logic. Hypothesis extension is still a limited set of clauses according to this method. And it is logically equivalent with the existing hypothesis extension. The experimental results and theoretical results are consistent.
\end{abstract}

\section{Introduction}

Common sense reasoning is reasoning for the limited information. Non-monotonic logic [1] is an effective method to deal with the reasoning by default. Therefore, it is the most important logic tool in common sense reasoning.

At present, there are two main methods to deal with the problems of non-monotonic logic in common sense reasoning. One method is the default logic and the other method is the belief revision method. There are many important research results about the field of the common sense reasoning. For the first method, McCarthy proposed the restrictive reasoning method [2-3]. In addition, Sandewall [4] proposed the method that it put the operator UNLESS in the first method. Later, the research about the monotonicity is becoming increasingly important in many fields. And it also achieved a lot of research results. For example, Reiter proposed the default logic [5]. McDermott and Doyle proposed the non-monotonic logic [6] and the related theory based on the modal logic [7]. A few years later, the equivalence relation between the default logic and the auto-epistemic logic [8] have been proved by Konolige. Until the mid of 1980s, Alchourron, Gardenfors and Makinsond proposed the famous theory of belief revision [9], it can also be named as AGM [10].

The belief revision method has been widely studied in common sense reasoning. However, some useful information may be lost or the result is unexpected during the process of revision. Therefore, the belief non-revision method [11] has been proposed. It can achieves the consistent set by limiting the process of reasoning. This method dose not change the original database.
This paper proposed a hypothesis extension algorithm based on the belief non-revision method. It provides the definition of hypothesis extension in propositional logic. The method of primitive implication was adopted in the algorithm. We will show that each hypothesis has the only extension. The experimental results demonstrate the process of reasoning is correct.

The remainder of the paper is structured as follows. In section 2, the theoretical basis are reviewed. Section 3 introduces the extension of hypothesis in propositional logic in detail and the results of experiment. Section 4 concludes the paper with a short discussion of future work.

\section{Theoretical basis}

\subsection{The definitions of the propositional logic}

In this paper, the hypothesis is limited to the propositional logic. The hypothesis extension is defined with the general resolution principle.

Definition 1 Proposition is a statement. It can determine the relationship described by the statement is either true or false.

Proposition can be divided into the atomic proposition and the complex proposition.

The atomic proposition is indecomposable. And it does not contain the logical connector.

$$
P, Q, R, \cdots, P_{i}, Q_{i}, R_{i}(i \geq 1)
$$

The logical connector is shown as the follows:

$$
\neg, \wedge, \vee, \rightarrow, \leftrightarrow
$$


The complex proposition consist of some atomic propositions by connecting with the logical connector, e.g., $(P \vee Q) \rightarrow \neg R$.

Definition 2 If $A$ is a formula, then $A$ must satisfies one of the following criteria:

(1) ' 0 ' and ' 1 ' are formulas.

(2) A atomic proposition is a formula.

(3) If $P, Q$ are formulas, then

$$
(\neg P),(P \wedge Q),(P \vee Q),(P \rightarrow Q) \text { and }(P \leftrightarrow Q)
$$

are formulas.

(4)The symbol string could be any limited combination of (1), (2)and (3). It is still a formula.

Definition 3 Literal is a atomic or a negation of atomic. A atomic and a negation of itself are collectively called a complementary pair.

Example $1 P, \neg P, \neg T$ are literals, $P, \neg P$ are a complementary pair.

Definition 4 Clause is the disjunctive form of finite literals.

Example $2 A=\neg P \vee Q \vee T \vee W \vee S$ is a clause.

\subsection{The resolution principle}

The resolution principle is inference rule. It means that a clause can be derived by two clauses.

Definition 5 For two clauses $S_{1}$ and $S_{2}$, there is a literal $l_{1}$ in the clause $S_{1}$, there is another literal $l_{2}$ in the clause $S_{2}$, if $l_{1}=\neg l_{2}$ then delete $l_{1}, l_{2}$ and connect the remaining part with the logical connector. The resolution of $S_{1}, S_{2}$ is denoted by $R\left(S_{1}, S_{2}\right)$.

Example 3 If $A, B$ are two clauses, and $C$ is the resolution of $A, B$, then $A \wedge B \rightarrow C$ is true. $R(A, B)$ is the result of resolution.

\subsection{The primitive implication}

In the 1950s, Quine [12] proposed a mechanized procedures. It can achieve the minimalist equivalent type of a formula. Actually, this is the original definition of the primitive implication. This problem is related to many fields of research. For example, the minimization of Boolean function [13], the update problem of logical database [14], finding the minimum support set in the truth maintenance system and so on.

Definition 6 Let $C$ be a clause in propositional logic. A set of all literals of $C$ is denoted by $\operatorname{literal}(C)$. And $\mid$ literal $(C) \mid$ is the cardinality of $C$.

Definition $7 C_{i}, C_{j}$ are two clauses. Both of them are not empty. If literal $\left(C_{i}\right) \subseteq \operatorname{literal}\left(C_{j}\right)$, then the clause $C_{j}$ subsume the clause $C_{i}$.
Definition 8 The primitive implication of a theory $T$ is donoted by $\forall P I(\Gamma)$. If a disjunctive form $C$ is the primitive implication of a theory $T$, then it must satisfies the following properties:

(1) $C$ is not a tautology.

(2) $T \mid=C$.

(3) There is not exist a clause $C^{\prime}$ such that $T \mid=C^{\prime}$ and $C^{\prime} \subseteq C$.

Definition 9 If $\Gamma$ is a consistent set of some clauses, then $\Gamma$ and $\forall P I(\Gamma)$ are logically equivalent. (the process of proof can be seen in [15])

\section{Hypothesis extension in propositional logic}

\subsection{The definition of hypothesis extension}

This paper gives an algorithm of hypothesis extension in propositional logic. In this section, the relative definitions are introduced.

Definition 10 Let $\Gamma=\left\{C_{1}, \cdots, C_{n}\right\}$ be a set of clauses in propositional logic. $C_{1}, \cdots, C_{n}$ are clauses. $\Gamma$ is a hypothesis and $\Gamma$ may be inconsistent.

Definition 11 Let $\Gamma$ be a hypothesis. $C$ is a clause but it is not empty. There is $C \in \operatorname{Con}(\Gamma)$. There is a finite sequence $C_{1}, \cdots, C_{n}$ satisfies the following properties:

(1)Let $C_{i}$ be a clause in the sequence. The clause $C_{i}$ satisfies the following conditions:

(1) $C_{i} \in \Gamma$.

(2) There are clauses $C_{i_{1}}, \cdots, C_{i_{k}}$ in front of $C_{i}$ in the sequence such that $C_{i_{1}} \wedge \cdots \wedge C_{i_{k}} \rightarrow C_{i}$ and $\left\{C_{i_{1}}, \cdots, C_{i_{k}}\right\}$ is consistent.

(2) $C=C_{i}$.

According to the definition, the attribute set $\operatorname{Con}(\Gamma)$ is a set of all results of reasoning.

Definition 12 Let $\Gamma$ be a hypothesis. If a clause is empty, then $\Gamma=\square$. The set of reconstruction is denoted by $R(\Gamma)$. For $\forall C \in R(\Gamma)$ should satisfies the following properties:

(1) $C \in \operatorname{Con}(\Gamma)$.

(2) $C \neq \square$.

(3)There is no clause $D \in \operatorname{Con}(\Gamma)$ such that $\mid$ lliteral $(R(C, D))|<|$ lliteral $(C) \mid$.

Theorem 1 If $\Gamma$ is a hypothesis, then the set of reconstruction $R(\Gamma)$ is consistent. (the details can be seen in [16]) 
Definition 13 Let $\Gamma$ be the set of some consistent formulas. $C_{i}$ is a formula in $\Gamma$. For $\forall P I(\Gamma), C_{i}$ should satisfies the following properties:

(1) $C_{i}$ is not a tautology.

(2) $\Gamma \mid=C_{i}$.

(3) There is not exist a formula $C_{j}$ and $C_{i} \subseteq C_{j}$ such that $\Gamma \mid=C_{j}$.

According to the above definitions, $P I(\Gamma)$ is the primitive implication of $\Gamma$.

Definition 14 If $\Gamma$ is a hypothesis, then the hypothesis extension is $E(\Gamma)=P I(R(\Gamma))$.

Example $4 \Gamma=\{p \vee q, \neg p \vee r, q \vee r \vee s, \neg r\}$ then

$$
\begin{aligned}
& R(\Gamma)=(\neg p, \neg r, q \vee s, q) \text { and } \\
& E(\Gamma)=P I((R(\Gamma))) \\
&=P I(\neg p, \neg r, q \vee s, q) \\
&=(\neg p, \neg r, q)
\end{aligned}
$$

\subsection{Hypothesis extension algorithm}

Actually, if $\Gamma$ is consistent, then $E(\Gamma) \Leftrightarrow P I(\Gamma)$. If $\Gamma$ is inconsistent, then $P I(\Gamma)$ is empty according to the definition of the primitive implication. However, $\Gamma$ may be inconsistent in this paper. Therefore, the process of hypothesis extension is divided into three steps. First, the attribute set $\operatorname{Con}(\Gamma)$ can be achieved by using the resolution principle. Second, the set of reconstruction $R(\Gamma)$ can be achieved by deleting the conflicts in Con $(\Gamma)$. Finally, due to $R(\Gamma)$ is consistent, we can achieve the hypothesis extension $E(\Gamma)=P I(R(\Gamma))$ according to the definition 14 .

$$
\Gamma \rightarrow \operatorname{Con}(\Gamma) \rightarrow R(\Gamma) \rightarrow E(\Gamma)
$$

But in the process of the resolution, we found that some operations are redundant because the results of different resolutions may be the same.

Therefore, the method Tison [17] have been adopted. This method is initially used for structure the primitive implication. It can optimizes the process of the resolution. And it also can improves the efficiency of the resolution.

The method Tison can be understood through the following example:

Example $6 \quad \Gamma=\{A \vee B, \neg B \vee C, \neg C \vee D\}$

The ordinary resolution:

1. $A \vee B$
2. $\neg B \vee C$
3. $\neg C \vee D$
4. $A \vee C \quad R(A \vee B, \neg B \vee C), B$
5. $\neg B \vee D \quad R(\neg B \vee C, \neg C \vee D), C$
6. $A \vee D \quad R(A \vee C, \neg C \vee D), C$
7. $A \vee D \quad R(A \vee B, \neg B \vee D), B$

The method Tision puts forwards a point. If the process of the resolution is executed according to the order of the literals, then the resolution can avoids redundancy (let the order be $A, B, D$ ).

The method Tison :

1. $A \vee B$

2. $\neg B \vee C$

3. $\neg C \vee D$

4. $A \vee C \quad R(A \vee B, \neg B \vee C), B$

5. $\neg B \vee D \quad R(\neg B \vee C, \neg C \vee D), C$

6. $A \vee D \quad R(A \vee C, \neg C \vee D), C$

Obviously, the steps of the method Tison is less than the steps of the ordinary resolution.

Algorithm As the following step shows:

The set of beliefs is denoted by $\Gamma$.

The literal set is denoted by literal $(\Gamma)$.

The attribute set is denoted by $\operatorname{Con}(\Gamma)$.

The set of reconstruction $R(\Gamma)$.

The hypothesis extension is denoted by $E(\Gamma)$.

1. $\Gamma \leftarrow\left\{C_{1}, \ldots, C_{n}\right\}, E(\Gamma)=\varnothing, R(\Gamma)=\varnothing$

2. foreach $C_{x}$ in $\Gamma$

3. if " $\vee " \in C_{x}$

4. $C_{x}=C_{x}-" \vee "$; literal $(\Gamma) \leftarrow C_{x}$

5. foreach $C_{x} \in \operatorname{literal}(\Gamma) ; C_{y} \in \operatorname{literal}(\Gamma)$

6. if $\neg C_{x} \cap C_{y} \neq \varnothing$

7. $\quad$ literal $(\Gamma)=$ literal $(\Gamma)-\left\{\neg C_{x}, C_{y}\right\}$

8. $\quad \Gamma \leftarrow C_{x}$, until literal $(\Gamma)=\varnothing$

9. foreach $C_{x} \in \Gamma ; C_{y} \in \Gamma$

10. $\quad$ if $x \in y$, then $\Gamma=\Gamma-\left\{C_{x}\right\}$

11. else, then $R\left(C_{x}, C_{y}\right) \rightarrow C_{z}$

12. $\quad$ if $C_{z} \in \Gamma$

13. $\Gamma=\Gamma-\left\{C_{z}\right\} ; \operatorname{Con}(\Gamma) \leftarrow C_{x}, C_{y}$

14. $\quad$ else $\operatorname{Con}(\Gamma) \leftarrow C_{x}, C_{y}, C_{z}$ 


$$
\begin{aligned}
& \text { 15. until } \Gamma=\varnothing \\
& \text { 16. } \quad R(\Gamma) \leftarrow \operatorname{Con}(\Gamma) \\
& \text { 17. } \quad \text { Let } \Gamma=R(\Gamma) \\
& \text { 18. repeat the step } 2 \text { to the step } 8 \\
& \text { 19. } R(\Gamma)=\Gamma \\
& \text { 20. return } E(\Gamma)=P I(R(\Gamma))
\end{aligned}
$$

\subsection{Experimental Case}

The process of the experiment as the following:

(1)The hypothesis is consistent and there are no inclusion relation in it:

$$
\begin{aligned}
& \Gamma=\{A \vee B, \neg B \vee C \vee D, \neg C \vee D\} \\
& \begin{array}{ll}
\text { 1. } A \vee B & \\
\text { 2. } A B \vee C \vee D & \\
\text { 3. } \neg C \vee D & \\
\text { 4. } A \vee C \vee D & R(A \vee B, \neg B \vee C \vee D), B \\
\text { 5. } \neg B \vee D & R(\neg B \vee C \vee D, \neg C \vee D), C \\
\text { 6. } A \vee D & R(A \vee B, \neg B \vee D), B \\
E(\Gamma)=\{A \vee B, \neg B \vee C \vee D, \neg C \vee D, A \vee C \vee D, \neg B \vee D, A \vee D\}
\end{array}
\end{aligned}
$$

(2)The hypothesis is inconsistent and there are no inclusion relation in it:

$$
\begin{array}{ll}
\Gamma=\{A \vee B, \neg B \vee C \vee D, \neg C \vee D, E, \neg E\} \\
\text { 1. } A \vee B & \\
\text { 2. } \neg B \vee C \vee D & \\
\text { 3. } \neg C \vee D & \\
\text { 4. } \neg E & \\
\text { 5. } E & R(A \vee B, \neg B \vee C \vee D), B \\
\text { 6. } A \vee C \vee D & R(\neg B \vee C \vee D, \neg C \vee D), C \\
\text { 7. } \neg B \vee D & R(A \vee B, \neg B \vee D), B \\
\text { 8. } A \vee D & R(\neg E, E), E \\
\text { 9. } \square &
\end{array}
$$$$
E(\Gamma)=\{A \vee B, \neg B \vee C \vee D, \neg C \vee D, A \vee C \vee D, \neg B \vee D, A \vee L
$$

(3) The hypothesis is consistent and the inclusion relation exist in it:

$$
\Gamma=\{A \vee B, \neg B \vee C \vee D, \neg C \vee D, C \vee D\}
$$

$$
\begin{aligned}
& \text { 1. } A \vee B \\
& \text { 2. } \neg B \vee C \vee D \\
& \text { 3. } \neg C \vee D \\
& \text { 4. } C \vee D \\
& \text { 5. } A \vee C \vee D \quad R(A \vee B, \neg B \vee C \vee D), B \\
& \text { 6. } \neg B \vee D \quad R(\neg B \vee C \vee D, \neg C \vee D), C \\
& \text { 7. } A \vee D \quad R(A \vee B, \neg B \vee D), B \\
& \text { 8. } D \quad R(\neg C \vee D, C \vee D), C \\
& E(\Gamma)=\{A \vee B, D\}
\end{aligned}
$$

(4) The hypothesis is inconsistent and the inclusion relation exist in it:

$$
\Gamma=\{A \vee B, \neg B \vee C \vee D, \neg C \vee D, C, \neg C\}
$$

1. $A \vee B$

$$
\begin{array}{ll}
\text { 2. } \neg B \vee C \vee D & \\
\text { 3. } \neg C \vee D & \\
\text { 4. } \neg C & \\
\text { 5. } C & \\
\text { 6. } A \vee C \vee D & R(A \vee B, \neg B \vee C \vee D), B \\
\text { 7. } \neg B \vee D & R(\neg B \vee C \vee D, \neg C \vee D), C \\
\text { 8. } \neg B \vee D & R(\neg B \vee C \vee D, \neg C), C \\
\text { 9. } \quad D & R(\neg C \vee D, C), C \\
\text { 10. } \quad A \vee D & R(A \vee B, \neg B \vee D), B \\
\text { 11. } \square & R(\neg C, C), C \\
E(\Gamma)=\{A \vee B, D\} &
\end{array}
$$

The experiment shows that the experimental result is consistent with the theoretical result. The algorithm obtains the good effect. It does not appear the situation that the important information is lost. In the process of experiment, we found that the working time will increases with the amount of data increases. However, this kind of situation is inevitable. In general, the algorithm is accurate and comprehensive.

\section{Conclusions}

The hypothesis extension has many good mathematical properties based on the non-revision method. The process of cognition is convergent in this paper. This nature shows that the non-revision method conforms to the process of cognition. And it is an effective solution to the non-monotonic reasoning in practice. In order to improve the application value of the non-revision method, this paper redefined the hypothesis extension based on the primitive implication. In the future, the research could be extended to the And/Or clause and the first-order logic.

\section{References}

1. Brewka, Gerhard, Non-monotonic reasoning: logical foundations of common sense, Cambridge University Press. (1991).

2. J. McCarthy, Circumscription a form of nonmonotonic reasoning, Artificial Intelligence, 13: 2739 (1980).

3. J. McCarthy, Applications of circumscription to formalize commonsense konwledge. Artificial Intellifence, 13: 7-39 (1986).

4. E. Sandewall, An approach to the frame Problem and its implementation. Machine Intelligence, 7: 195-204 (1972).

5. R. Reiter, A logic for default reasoning, Artificial Intelligence, 13: 81-132 (1980).

6. D. McDermott, J. Doyle, Non-monotonic logic. Artificial Intelligence, 13: 41-72 (1980).

7. C. Mihir, G. Suiata, Non-monotonic Logics and Algebras, Journal of Chongqing University of Posts and Telecommunications (Natural Science Edition), 20: 355-360 (2008).

8. D.M. Gabbay, Heoretical foundations for nonmonotonic reasoning in expert systems. in: K. R. Apt 
(Ed.), Proceedings NATO Advanced Study Institute on Logics and Models of Concurrent Systems, Springer, Berlin, 439-457 (1985).

9. C.E. Alchourrón, D. Makinson, On the logic of theory change: Safe contraction, Symbolic Logic, 405-422 (1985).

10. A. Darwiche, J. Pearl, On the logic of iterated belief revision, Artificial Intelligence, 1-29 (1997).

11. Y. Shang, A.S. Deng, X.D. Jiu, The Quantitative Correction Method of Inconsistent belief meet the AGM public discussion, Computer Engineering and Science, 26: 106-109 (2004).

12. W.V. Quine, The problem of simplifying truth functions. American Mathematical Monthly, 521-531 (1952).

13. P. Tison, Generalization of consensus theory and application to the minimization of boolean functions. Electronic Computers, IEEE.

14. J. Pais, P. Jackson, Partial monotonicity and a new version of the Ramsey test. Studia Logica, 51(1): 2147 (1992).

15. M.A. Falappa, Explanations belief revision and defeasible reasoning. Artificial Intelligence, 141: 128 (2002).

16. J.X. Zhang, The research about inconsistent beliefs quantitative a modified hierarchical. Dalian, Dalian Maritime University (2013).

17. P. Tison, Generalization of consensus theory and application to the minimization of boolean functions. Electronic Computers, 4: 446-456 (1967). 